\title{
Dual-Use Intraoperative MRI in Glioblastoma Surgery: Results of Resection, Histopathologic Assessment, and Surgical Site Infections
}

\author{
Johannes Wach ${ }^{1}$ Claudia Goetz ${ }^{2}$ Kasra Shareghi ${ }^{2}$ Torben Scholz ${ }^{2,3}$ Volker Heßelmann ${ }^{4}$ \\ Ann-Kathrin Mager ${ }^{4}$ Joachim Gottschalk ${ }^{5}$ Hartmut Vatter $^{1}$ Paul Kremer $^{2}$
}

\footnotetext{
${ }^{1}$ Department of Neurosurgery, Universitatsklinikum Bonn Zentrum fur Nervenheilkunde, Bonn, North Rhine Westphalia, Germany

${ }^{2}$ Department of Neurosurgery, Asklepios Kliniken Hamburg GmbH Asklepios Klinik Nord Standort Heidberg, Hamburg, Hamburg, Germany

${ }^{3}$ Department of Neurosurgery, Medical Center University of Aachen, Aachen, NRW, Germany

${ }^{4}$ Department of Neuroradiology, Asklepios Kliniken Hamburg GmbH Asklepios Klinik Nord Standort Heidberg, Hamburg, Hamburg, Germany

${ }^{5}$ Department of Neuropathology, Asklepios Kliniken Hamburg GmbH Asklepios Klinik Nord Standort Heidberg, Hamburg, Hamburg, Germany
}

\begin{abstract}
Address for correspondence Johannes Wach, MD, Department of Neurosurgery, University-Hospital Bonn, Sigmund-Freud-Straße-25, Bonn 53127, Germany (e-mail: Johannes.Wach@ukbonn.de).
\end{abstract}

J Neurol Surg A 2019;80:413-422.

\section{Abstract \\ Keywords \\ - antibiotic prophylaxis \\ - extent of resection \\ - intraoperative MRI \\ - surgical site infections \\ - glioblastoma \\ - histopathology}

Background To achieve maximal resection in glioblastoma (GBM) surgery, intraoperative imaging is important. An intraoperative magnetic resonance imaging (iMRI) suite used for both diagnostic and intraoperative imaging is considered being a reasonable concept for modern hospital management. It is still discussed if the dual use increases the risk of surgical site infections (SSI). This article assesses the rate of gross total resection (GTR), extent of resection (EOR), and histopathology after iMRIguided resections in patients with GBM. The rate of surgical site infections (SSIs) is evaluated.

Methods In all, 79 patients with GBM were operated on with iMRI. Additional resection was performed if $\mathrm{iMRI}$ depicted contrast enhancing tissue suggestive of residual tumor. GTR and EOR were determined by segmentation and volumetric analysis of the MR images. SSIs and the role of intravenous only or intravenous plus intrathecal antibiotics were evaluated. Statistical analysis was performed to detect the sensitivity, specificity, positive predictive value, and negative predictive value of iMRIguided extended resections. Pearson's two-tailed chi-square test was performed to evaluate the rates of GTR and variables associated with SSI.

Results GTR was achieved in 59 patients (74.68\%). Rate of GTR was $35.44 \%$ before iMRI and additional resections $(p<0.0001)$. Mean EOR was $96.27 \%$. Positive predictive value for tumor cells in the additionally resected tissue was $88.6 \%$, negative predictive value was $100 \%$, sensitivity was $100 \%$, and specificity was $70.6 \%$. Rate of SSIs was $5.06 \%$ $(n=4)$. Two superficial SSIs, one subdural empyema and one cerebritis, were seen. received

January 21, 2019

accepted after revision

March 4, 2019

published online

July 4, 2019
(C) 2019 Georg Thieme Verlag KG Stuttgart · New York
DOI https://doi.org/

10.1055/s-0039-1692975. ISSN 2193-6315. 
SSI rates with parenteral only and additional intrathecal antibiotics were $0 \%$ and $8 \%$, respectively $(p=0.133)$.

Conclusion Increase of extent of tumor resection using iMRI is evident. SSI rate is within the normal range of neurosurgical procedures. A dual-use iMRI suite is a safe concept.

\section{Introduction}

Reducing tumor volumes as much as possible and enabling good neurologic outcomes comprise a major task in neurosurgery. An extent of resection (EOR) $>98 \%$ is accepted as a positive prognostic factor for survival of glioblastoma (GBM) patients. ${ }^{1,2}$ Reduction of tumor volumes as much as possible is also recommended for low-grade gliomas. ${ }^{3}$ Moreover, it is also important in the surgical treatment of pituitary adenomas to achieve a gross total resection (GTR) because it is known to decrease the rate of recurrence. ${ }^{4}$ Intraoperative imaging such as fluorescence, ultrasound, or intraoperative MRI (iMRI) are powerful tools to determine the EOR of those diseases.

The second task that must be considered in the surgical treatment of intracranial lesions is compensating for brain shift that depends on tumor size, localization of tumor, surrounding brain edema, positioning of the patient, type of neurosurgical approach, administration of osmotic diuretics, and the opening of the ventricular system. ${ }^{5}$ Mean brain shift of tumor margins, sulci, third ventricle, and lateral ventricles has been observed from 6 to $9 \mathrm{~mm}$. ${ }^{6}$ Using iMRI in combination with neuromonitoring and mapping represents an excellent tool to achieve maximal surgical results and ensure a good postoperative neurologic outcome. Furthermore, with iMRI, neuronavigation can be updated to compensate for brain shift. Despite the advantages of iMRI, it is not yet universally established because of high purchase price and operating costs. Furthermore, it is suggested that an iMRI in dual use causes a higher infection rate, and recent data estimated the cost for surgical site infection (SSI) after craniotomy to be $£ 9,283$. $^{7}$

To perform neurosurgical procedures in an iMRI suite, excellent hygiene must be maintained to ensure low rates of SSI. Generally, 5\% is the accepted threshold for SSI in neurosurgery. ${ }^{8}$ A 2017 study including 132,063 patients showed that the postoperative infection rate in neurosurgical procedures is $5.3 \%{ }^{9}$ Because of highly varying surgical conditions, there are also reports of SSI rates ranging between $1 \%$ and $11 \%{ }^{7,10-14}$

Due to increasing rates of SSI caused by multiresistant bacteria, use of perioperative antibiotic prophylaxis is highly debated. Recent data showed that lincosamides, glycopeptides, third-generation cephalosporins, other combinations of prophylactic antibiotics, or antibiotics in the penicillin family alone are better prophylaxis against SSI than firstgeneration cephalosporins among neurosurgical patients, ${ }^{15}$ indicating a need to cover multiresistant and gram-negative bacteria. The use of vancomycin is still controversial because of its adverse effects and increasing rates of vancomycinresistant enterococci. ${ }^{16}$ Other studies of shunt surgery and studies in which vancomycin was administered in the subgaleal space reported a beneficial outcome. ${ }^{17,18}$ We assessed the clinical routine in our iMRI suite that is in dual use for both diagnostic and intraoperative neurosurgical imaging. We focused on analyzing the success of surgery, evaluating the resection rates, histopathologic accuracy of iMRI-guided resections, safety of the iMRI suite concerning SSI, and cefazolin only versus additional intrathecal administration of vancomycin and gentamicin antibiotic prophylaxis in all GBM procedures performed in the dual-use iMRI suite.

\section{Methods}

\section{Study Design}

All data were analyzed retrospectively. Surgeries were performed between July 1, 2011, and June 31, 2015. Primary, secondary, recurrent, and irradiated GBMs were included in the group of 79 GBM patients. Ethical approval was received by a university committee. Specific consent of the patients to take part in this retrospective study was not necessary. All personal data of patients were anonymized.

\section{Intraoperative Imaging Techniques}

\section{5-Aminolevulinic Acid}

5-Aminolevulinic (5-ALA) was administered orally as a single dose ( $20 \mathrm{mg} / \mathrm{kg}$ body weight) an average of 3 hours (range: 2-5 hours) preoperatively in 68 GBM patients. For visualization of the fluorescence, we used the PENTERO 600 microscope (Zeiss, Oberkochen, Germany) with an integrated head-up display to visualize the neuronavigation and a filter to visualize $405 \mathrm{~nm}$ fluorescence.

\section{Intraoperative MRI and Corresponding Equipment}

All examinations were performed in a 1.5-T iMRI (Philips Diamond Select Achieva 1.5 T; Philips, Amsterdam, Netherlands) with the administration of $0.1 \mathrm{mmol}$ GD-DTPA $/ \mathrm{kg}$ body weight when enhancing tumor tissue was seen in the preoperative MR images. The iMRI suite (-Fig. 1) allows a dual use of the MR system.

The system can be shared for daily diagnostic use for inpatients and outpatients, and for intraoperative imaging because of the separate entrance through double doors from the operating room. On the day of surgery the iMRI suite is reserved for 3 hours for the intraoperative imaging procedure. The iMRI suite gets cleaned like a regular surgical suite the evening before and also 1 hour before iMRI scanning. The MR suite is equipped with operative air conditioning that has 


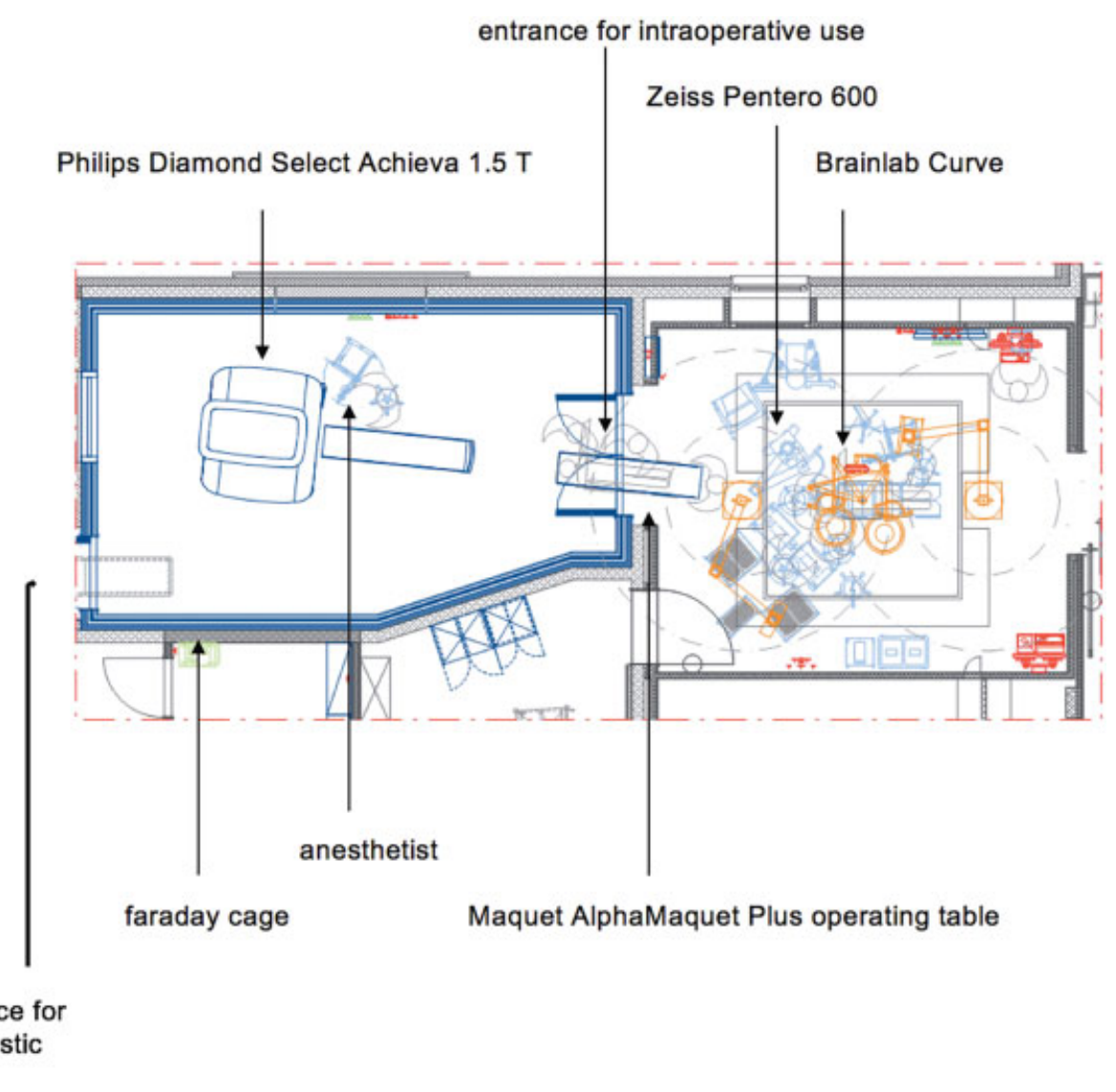

Fig. 1 Drawing of the layout of the intraoperative magnetic resonance imaging suite.

to be started 30 minutes before intraoperative scanning begins. A sterile cover of the patient is essential for the whole procedure to maintain hygienic conditions. Consequently, before skin incision one sterile cover has to be applied above the head. After the conventional resection with neuronavigation and 5-ALA, a second sterile cover is placed on the patient before the upper head coil of the MRI is integrated into the skull clamp. Afterward, the whole setup is wrapped in a sterile cover reaching over the head and below the shoulders (-Fig. 2). For the sterile cover, positioning and transport in and out of the iMRI one hour is calculated. After the whole procedure is finished, the iMRI suite gets cleaned again before diagnostic imaging is continued.

All patients were registered on the neuronavigation system (Brainlab Curve, Brainlab, Feldkirchen, Germany) using preoperative T1 magnetization-prepared rapid acquisition with gradient echo (MPRAGE) scans and a Softouch registration pointer (Brainlab, Feldkirchen, Germany). Patients' heads were fixed in position with the Noras Head Holder-kit Heidberg (Noras, Würzburg, Germany). The Alpha Maquet Plus (MRcompatible table)(Maquet, Rastatt, Germany) was used during surgery and for transporting the patient into the MR suite.

\section{Surgical Procedure}

-Fig. 3 shows our surgical workflow for GBM surgery. Initially, a white-light resection under neuronavigation guidance was performed. When the surgeon assumed that GTR of the tumor was achieved, hemostasis followed. Afterward the resection cavity was examined using 5-ALA, and areas suspicious for remaining tumor tissue were resected. Then the patient was transported into the iMRI according to a specific protocol and controlled by a physician using a checklist. After completing the iMRI scan, images were evaluated by the neuroradiologist and neurosurgeon concerning residual contrast enhancement. If no residual enhancement was visible, surgery was finished. In all remaining cases with residual enhancement, the resection cavity was reexamined by re-referencing the neuronavigation using the intraoperative MR images. If suspicious tumor was in a noneloquent area, iMRI-guided resection was performed.

\section{Volumetric Segmentation and Definition of GTR and EOR}

Volumetric segmentation of GBMs was performed using Aquarius intuition software (Terarecon, Houston, Texas, United States). We analyzed contrast-enhanced T1-weighted 1.5-T MRI scans with a semiautomatic volumetric tumor segmentation algorithm (-Fig. 4). Pre-, intra-, and postoperative (obtained within 72 hours after surgery) MRI scans were examined.

GTR was defined as removal of $\geq 95 \%$ of enhancing tissue and the loss of nodular enhancement, and subtotal resection as the removal of $<95 \%$ of the enhancing tumor tissue. EOR was determined by comparing preoperative and postoperative (obtained within 72 hours after surgery) tumor volumes on MRI scans. 


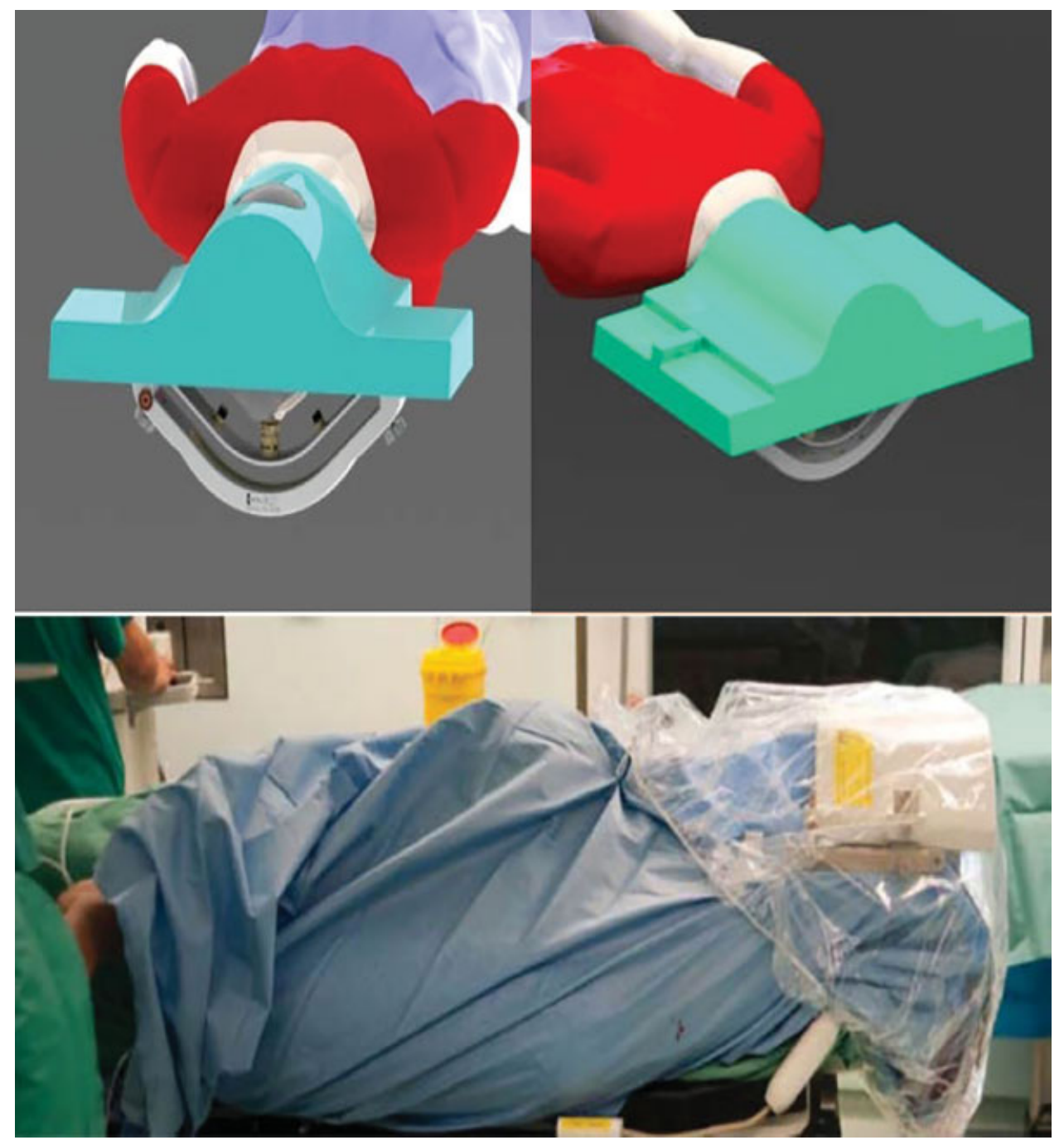

Fig. 2 Sterile cover of a patient transported into the intraoperative magnetic resonance imaging suite.

\section{Histologic Analysis}

Histologic evaluation was performed according to the World Health Organization 2007 diagnostic consensus criteria. ${ }^{19}$ For this purpose, paraffin sections were stained with hematoxylin and eosin. Sections were examined immunohistochemically with MIB-1 antibody, GFAP, and IDH1. MGMT status was determined by methylation-specific polymerase chain reaction.

\section{Antibiotic Prophylaxis}

Perioperative antibiotic prophylaxis was used in 79 patients and in two different combinations. One group of 50 patients received only cephalosporins ( $2 \mathrm{~g}$ cefazolin or $1.5 \mathrm{~g}$ cefuroxime) intravenously at anesthesia introduction. Another group of 29 patients received in addition to the intravenous cephalosporin a single intrathecal antibiotic prophylaxis composed of vancomycin $(20 \mathrm{mg})$ and gentamicin $(5 \mathrm{mg})$ shortly before closing the dura mater. The intrathecal antibiotic prophylaxis was injected into the resection cavity at the end of the operation after completion of hemostasis, shortly before the closure of the dura.
The additional administration of vancomycin and gentamicin was part of a change of the perioperative antibiotic prophylaxis protocol in our department after the introduction of the MRI in 2011. Patients with risk factors associated with SSI (diabetes mellitus, obesity, long-term corticosteroid use, recently received radiotherapy or chemotherapy) and the absence of acknowledged contraindications for vancomycin and gentamicin were candidates for the intrathecal antibiotic prophylaxis.

\section{Definition of Surgical Site Infections}

SSIs were identified based on postoperative clinical notes with the use of the Centers for Disease Control and Prevention definition of infection (infection involving superficial incisional, deep incisional, and/or organ/space SSI) ${ }^{20}$ Definition of SSI was not limited to a period of 30 days after surgery. Infections diagnosed at any time in the follow-up period were included. Wound dehiscences without evidence of infection were not considered as an SSI. Superficial SSIs were defined as infections affecting the cutis and subcutis and caused by cutaneous bacteria. Deep SSIs included 


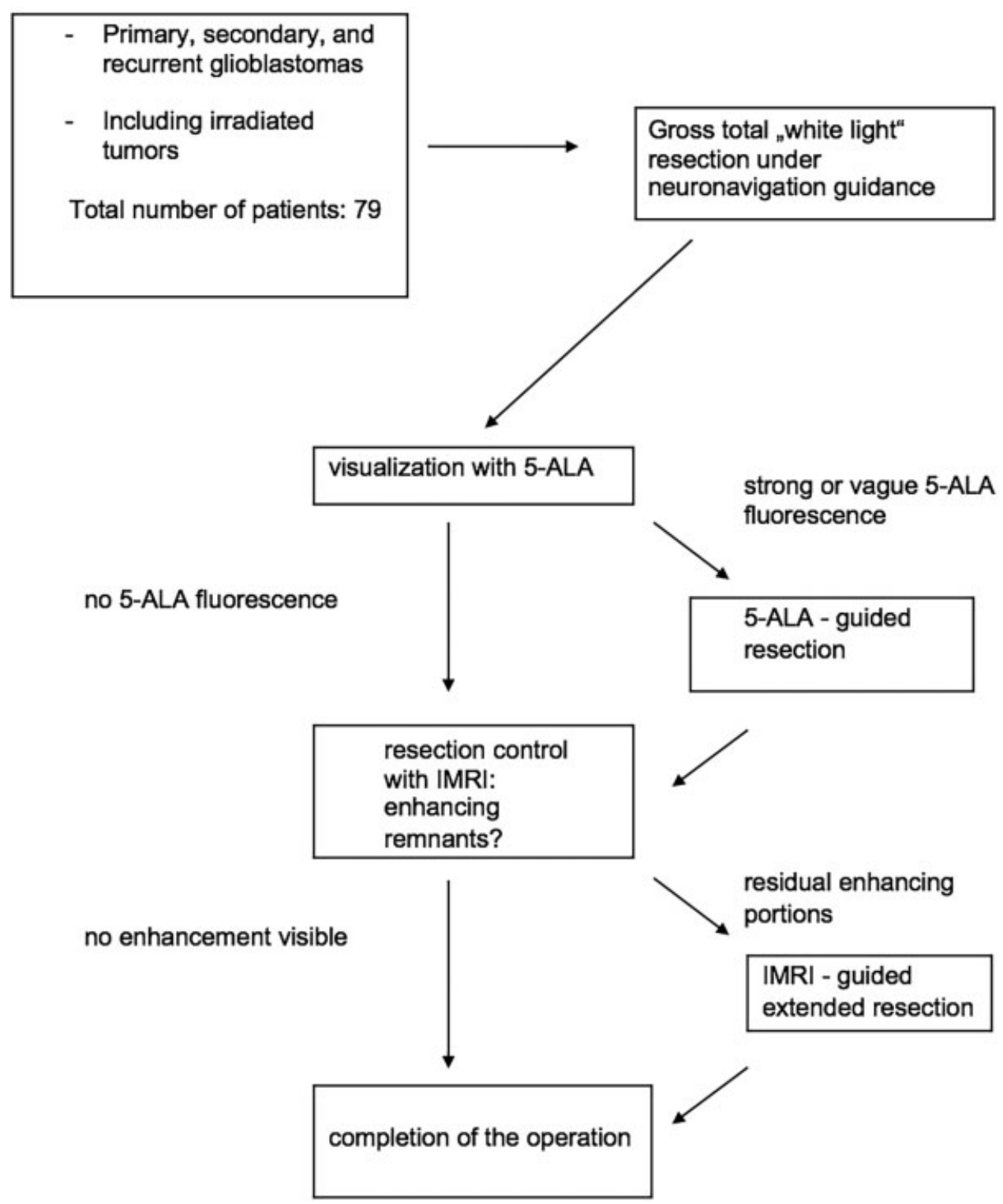

Fig. 3 Surgical workflow. 5-ALA, 5-aminolevulinic acid; iMRI, intraoperative magnetic resonance imaging.

meningitis, brain abscess, subdural empyema, and cerebritis. The following parameters were examined in patients with SSI: symptoms, risk factors for SSI, evidence of specific bacteria from cultures, cerebrospinal fluid parameters, C-reactive protein, and white blood cell count. To evaluate the etiology of SSIs, we analyzed preexisting illness, age, sex, scanning time, need for additional resections, previous surgery with craniotomy, perioperative antibiotic prophylaxis, and causative agent.

\section{Statistical Methods}

Descriptive and exploratory methods were used to analyze the data. We calculated mean values, standard error of the mean, and performed chi-square tests. Missing data were not included in our calculations. We calculated positive predictive value (PPV), negative predictive value (NPV), and sensitivity and specificity of iMRI to detect tumor tissue. Confidence intervals (CIs) were calculated to assess the necessity of additional resections to achieve GTR. Pearson's chi-square tests (two sided) were performed to compare the two kinds of antibiotic prophylaxis, age, sex, previous surgery with craniotomy, and risk factors (diabetes mellitus, obesity, long-term corticosteroid use, recently received radiotherapy or chemotherapy) concerning the incidence of SSI. Student two-sample $t$ tests were conducted to analyze mean values. For all statistical tests and graphics, we used SPSS v.23 (IBM Corp., Armonk, New York, United States) and Microsoft Excel 2016 (Microsoft Corp., Redmond, Washington, United States).

\section{Results}

\section{Characteristics of Patients with Glioblastomas}

In all, 79 surgical procedures in patients with GBM were performed within the iMRI suite. - Table 1 shows the general characteristics of the GBM patient collective. Most of the 


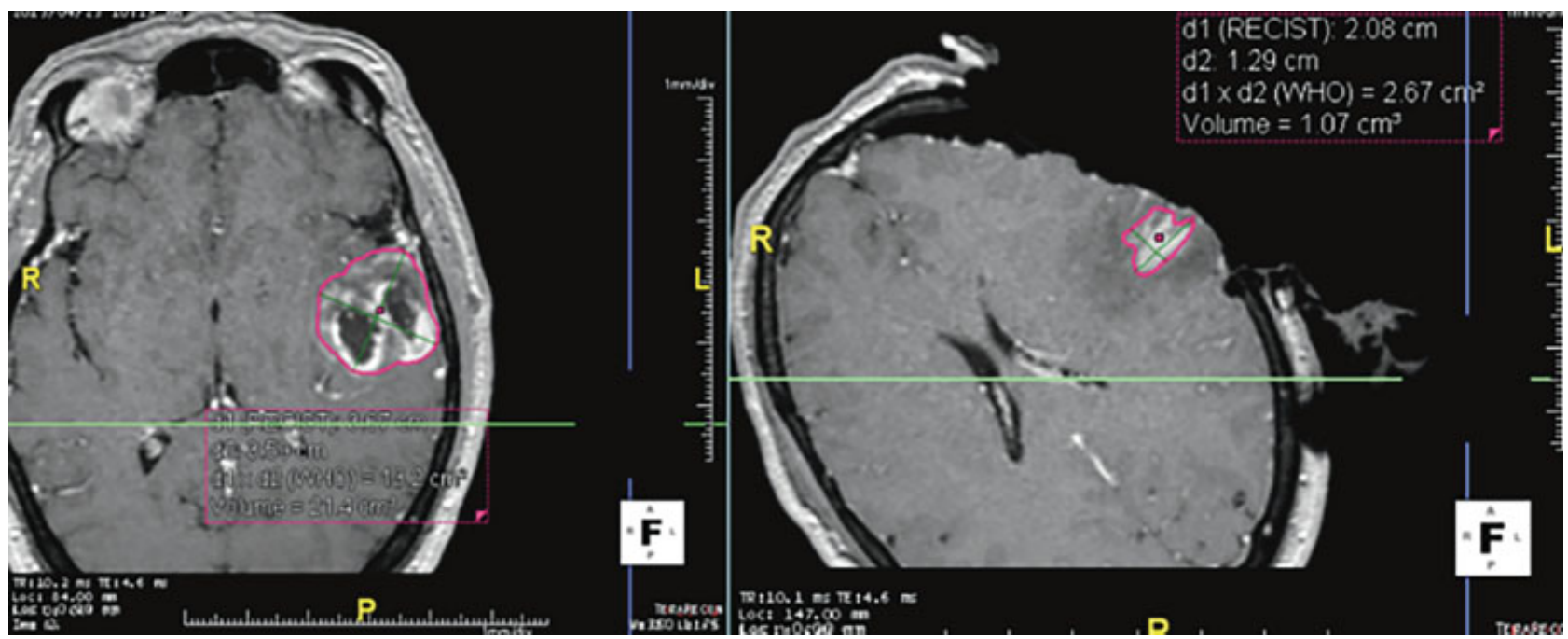

Fig. 4 Volumetric segmentation of preoperative and intraoperative contrast-enhanced T1-weighted magnetic resonance imaging scans.

patients had primary GBMs The rate of tumors located in eloquent areas was quite high $(45.6 \%)$. All patients showed the typical, strong, peripheral ring gadolinium (Gd) enhancement in preoperative MRI scans. In all, 68 patients received 5-ALA before surgery, and 58 of them showed a strong intraoperative fluorescence; therefore, the correlation

Table 1 Patient characteristics

\begin{tabular}{|l|l|l|}
\hline $\begin{array}{l}\text { GBM patient } \\
\text { characteristics } \\
(\boldsymbol{n}=\mathbf{7 9 )}\end{array}$ & & \\
\hline Characteristic & & \\
\hline Male-to-female ratio & & $1.82: 1$ \\
\hline Mean age, $\mathrm{y}$ & & $58.6(\mathrm{SD} \pm 12.1)$ \\
\hline Mean MIB-I value & & $32.6(\mathrm{SD} \pm 17.6)$ \\
\hline $\begin{array}{l}\text { IDH1 point } \\
\text { mutation (\%) }\end{array}$ & & $8(10.8)$ \\
\hline $\begin{array}{l}\text { MGMT promoter } \\
\text { hypermethylation (\%) }\end{array}$ & & $20(25.3)$ \\
\hline \begin{tabular}{l} 
Affected lobe (\%) \\
\cline { 2 - 3 }
\end{tabular} & Temporal & $28(35.4)$ \\
\hline & Frontal & $21(26.6)$ \\
\hline & Parietal & $16(20.3)$ \\
\hline & Occipital & $6(7.6)$ \\
\cline { 2 - 3 } & Multilobular & $8(10.1)$ \\
\hline Left-sided lesions (\%) & & $37(46.8)$ \\
\hline Eloquent areas (\%) & & $36(45.6)$ \\
\hline $\begin{array}{l}\text { Mean tumor } \\
\text { volume, cm }\end{array}$ & & 29.43 \\
\hline $\begin{array}{l}\text { No. of additional } \\
\text { resections } \\
\text { after iMRI (\%) }\end{array}$ & & $55(69.6)$ \\
\hline
\end{tabular}

Abbreviations: GBM, glioblastoma; IDH1, isocitrate dehydrogenase; iMRI, intraoperative magnetic resonance imaging; MGMT, O6-methylguanin-DANN methyltransferase; MIB-I, Molecular Immunology Borstel; $\mathrm{SD}$, standard deviation. between Gd enhancement on preoperative T1-weighted contrast-enhanced MRI and positive 5-ALA fluorescent reaction was $85.29 \%$.In all cases we performed an iMRI only once.

\section{Surgical Success with iMRI-guided Glioblastoma Resection}

Extent of Resection

EOR was determined in 74 of 79 patients. Five cases were excluded due to low and/or diffuse contrast-enhancing properties in pre- or postoperative MRI scans making reliable segmentation of T1-weighted contrast-enhanced MR images nearly impossible, as judged by a neuroradiologist. The mean EOR was $96.27 \%$. Table 2 summarizes the determined tumor volumes.

Rate of Subtotal and Gross Total Resection Before and After Extended Resections

GTR was determined at two different time points. At the time of iMRI, GTR was found in 28 patients (35.44\%). After iMRI and additional resection, a GTR was achieved in 59 of the 79 GBM patients $(74.68 \% ; p<0.0001)$. Thus, iMRI helped to achieve GTR in additional 31 patients: 52.54\% of all patients with a GTR in the postoperative MRI benefited from iMRI-guided additional resections. - Fig. 5 shows the increase in surgical success using iMRI. The $\mathrm{CI}$ to achieve GTR by iMRI-guided extended resection was also calculated (95\% CI, 0.56-0.84).

Histopathology of iMRI-guided Additional Resections In 55 of 79 (69.6\%) GBM operations, we performed additional resections because iMRI showed residual Gd enhancement. The PPV, that the contrast enhancement in iMRI corresponds to tumor, was $88.6 \%$. In the specimens of 45 of 55 patients in whom iMRI-guided additional resections were performed, tumor tissue could be confirmed. Sensitivity was $100 \%$ and specificity was $70.6 \%$. The NPV was $100 \%$ - Table 3 shows the histopathologic assessment. 
Table 2 Determined values of volumetric tumor segmentation

\begin{tabular}{|l|l|}
\hline Volumetric segmentation & iMRI \\
\hline Variables & \\
\hline $\begin{array}{l}\text { Mean preoperative tumor } \\
\text { volume, } \mathrm{cm}^{3}\end{array}$ & 29.43 \\
\hline Range & $1.16-121$ \\
\hline SEM & 25.8 \\
\hline $\begin{array}{l}\text { Mean intraoperative tumor } \\
\text { volume, cm }\end{array}$ & 5.3 \\
\hline $\begin{array}{l}\text { Mean postoperative tumor } \\
\text { volume, cm }\end{array}$ & 0.565 \\
\hline Range & $0-5.97$ \\
\hline SEM & 1.67 \\
\hline Mean EOR, \% & 96.27 \\
\hline Range & $16.56-100$ \\
\hline SEM & 11.04 \\
\hline
\end{tabular}

Abbreviations: EOR, extent of resection; iMRI, intraoperative magnetic resonance imaging; SEM, standard error of the mean.

\section{Surgical Site Infections}

The mean duration of the scanning time in the iMRI was 33.6 $( \pm 7.17)$ minutes. Four of the 79 patients (5.06\%) had an SSI. There were two superficial SSIs, one subdural empyema and one cerebritis ( - Table 4 ). Mean duration of the scanning time in cases with an SSI was 29 minutes and failed to be a statistically significant variable. Overall, 28 (35.44\%) of the patients had a previous craniotomy and were treated because of recurrence. SSI was observed in two (7.14\%) of the recurrent gliomas. Two of the four patients with SSI had risk factors such as prior radiochemotherapy and morbid obesity. In two of the four patients, we performed an extended resection after iMRI. - Table 5 shows the cases with SSIs in detail.

The correlation between SSI and administered antibiotic prophylaxis was examined in the 79 patients. In 50 patients who received only intravenous antibiotic prophylaxis (cefazolin or cefuroxime), four SSIs (8\%) occurred. In the second group of 29 patients who received additional intrathecal
Table 3 Histopathologic analyses of iMRI-guided extended resections of glioblastoma

\begin{tabular}{|l|c|}
\hline Histopathologic assessment, \% & \\
\hline Sensitivity & 100 \\
\hline Specificity & 70.6 \\
\hline Positive predictive value & 88.6 \\
\hline Negative predictive value & 100 \\
\hline
\end{tabular}

Abbreviation: iMRI, intraoperative magnetic resonance imaging.

Table 4 Overview of surgical site infections

\begin{tabular}{|l|l|}
\hline Overall rate of SSIs & $4(5.06 \%)$ \\
\hline Superficial SSI & $2(2.53 \%)$ \\
\hline Subdural empyema & $1(1.27 \%)$ \\
\hline Cerebritis & $1(1.27 \%)$ \\
\hline
\end{tabular}

Abbreviation: SSI, surgical site infection.

antibiotic prophylaxis (vancomycin and gentamicin), no SSIs were observed ( $p=0.133$ ). Age, sex, risk factors in medical history, previous surgery, preoperative tumor volumes, duration of surgery, and need of additional resections were also analyzed and not statistically significant concerning the rate of SSI ( - Tables 6 and 7 ).

\section{Discussion}

Despite continuous improvement in neurosurgical techniques, GBM still represents a fatal diagnosis. Complete tumor resection (EOR $>98 \%$ ) is the major goal. 5-ALA fluorescence and iMRI are the two most efficient approaches to achieve a GTR. However, iMRI, in particular, is not yet established because of its poor cost-benefit ratio if only used intraoperatively. Therefore, we analyzed our dual-use iMRI suite concerning results of surgical resection, histopathologic accuracy, and SSI in GBM cases.

In our study, $85.29 \%$ of the Gd-enhancing GBMs showed a strong 5-ALA fluorescence intraoperatively. This finding supports the hypothesis that not only a disrupted blood-brain

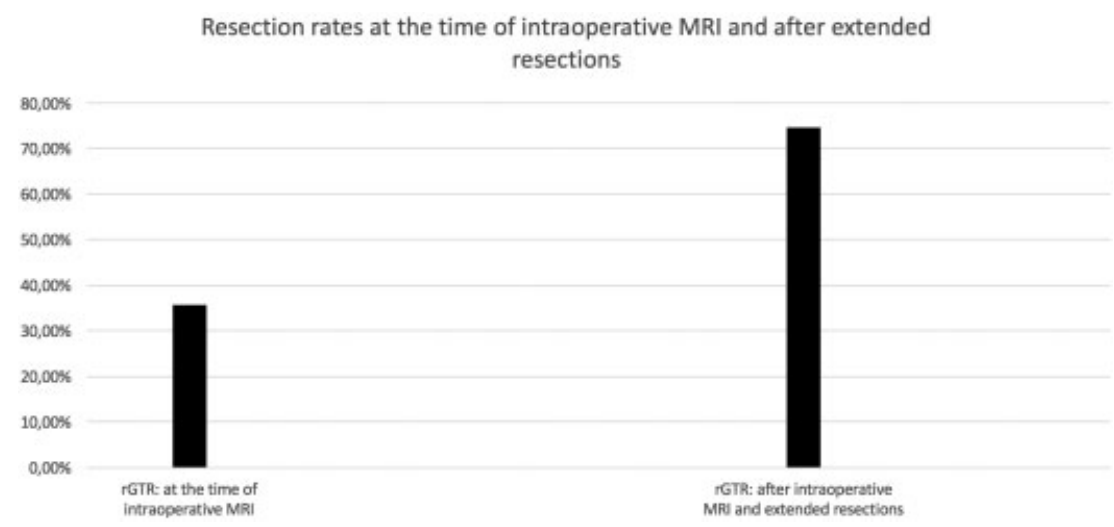

Fig. 5 Rate of gross total resection: before and after interoperative magnetic resonance imaging (MRI)-guided additional resections. 
Table 5 Details of patients with surgical site infections

\begin{tabular}{|l|l|l|l|l|l|l|l|}
\hline & \multicolumn{2}{|l|}{ Cases with SSI } & $\begin{array}{l}\text { Pathogenic } \\
\text { bacteria } \\
\text { Age, y/Sex }\end{array}$ & $\begin{array}{l}\text { Infection } \\
\text { prophylaxis }\end{array}$ & $\begin{array}{l}\text { No. of } \\
\text { additional } \\
\text { resections }\end{array}$ & $\begin{array}{l}\text { Second } \\
\text { surgery } \\
\text { for recurrent } \\
\text { glioma }\end{array}$ & $\begin{array}{l}\text { Duration of } \\
\text { iMRI scan, min }\end{array}$ \\
\hline $65 / \mathrm{M}$ & Sf SSI & $\begin{array}{l}\text { Staphylococcus } \\
\text { epidermidis }\end{array}$ & $2 \mathrm{~g}$ cefazolin & 1 & Yes & $\begin{array}{l}\text { Previous } \\
\text { radiotherapy } \\
\text { and chemotherapy }\end{array}$ & 20 \\
\hline $65 / \mathrm{F}$ & Sf SSI & $\begin{array}{l}\text { Enterobacter } \\
\text { cloacae }\end{array}$ & $2 \mathrm{~g}$ cefazolin & 0 & No & - & 33 \\
\hline $54 / \mathrm{M}$ & $\begin{array}{l}\text { Subdural } \\
\text { empyema }\end{array}$ & $\begin{array}{l}\text { Staphylococcus } \\
\text { aureus }\end{array}$ & $2 \mathrm{~g}$ cefazolin & 0 & No & Morbid obesity & 30 \\
\hline $58 / \mathrm{M}$ & Cerebritis & $\begin{array}{l}\text { Pseudomonas } \\
\text { aeruginosa }\end{array}$ & $2 \mathrm{~g}$ cefazolin & 1 & Yes & - & 30 \\
\hline
\end{tabular}

Abbreviations: F, female; iMRI, intraoperative magnetic resonance imaging; M, male; Sf, superficial.

Table 6 Univariate analysis of risk factors for SSI

\begin{tabular}{|l|l|l|l|}
\hline Variables & Rates of SSI & Rates of SSI & $p$ Value \\
\hline Age, y (\%) & $>$ 65: 0/20 (0) & $<65: 4 / 59(6.8)$ & 0.23 \\
\hline Sex (\%) & Female: 1/28 (3.6) & Male: 3/51 (5.9) & 0.654 \\
\hline Risk factors (\%) & Yes: 2/16 (12.5) & No: 2/63 (3.2) & 0.129 \\
\hline Second surgery for recurrent glioma (\%) & Yes: 2/28 (7.14) & No: 2/51 (3.9) & 0.532 \\
\hline iMRI-guided additional resections (\%) & Yes: 2/55 (3.6) & No: 2/24 (8.3) & 0.381 \\
\hline Duration of surgery, h (\%) & $\geq 4$ h: 2/56 (3.6) & h: $2 / 23(8.7)$ & 0.345 \\
\hline Perioperative antibiotic prophylaxis (\%) & $\begin{array}{l}\text { Parenteral plus } \\
\text { intrathecal antibiotics: } 0 / 29(0)\end{array}$ & Parenteral antibiotics: 4/50 (8) & 0.133 \\
\hline
\end{tabular}

Abbreviations: iMRI, intraoperative magnetic resonance imaging; SSI, surgical site infection.

barrier is responsible for the fluorescence. The decreased activity of the enzyme ferrochelatase seems to play an important role. ${ }^{21}$ However, in 11 cases we did not use 5-ALA because of contraindications concerning other drugs, elevated liver enzymes, or brain metastases suspected preoperatively. GTR was achieved in $74.68 \%$ of all GBM cases. This corresponds to results by Roder et al, ${ }^{22}$ who compared GTR in three different groups. The rate of GTR in their iMRI group was $74 \%$, in the 5-ALA group $46 \%$, and in the white-light group $28 \%$. Other studies made a preoperative selection as to whether GTR could be achieved, resulting in a GTR rate of $96 \%$ with high-field iMRIguided resections in non-eloquent tumor locations. ${ }^{23}$ A larger study consisting of 139 patients showed a rate of GTR with 5-ALA-guided resections of $65 \%{ }^{24}$ Our GTR rate of $74.68 \%$ achieved by 5 -ALA and iMRI is relatively high considering that the rate of tumors located in eloquent areas was $45.57 \%$ and that we did not select for the ability to achieve GTR. Therefore, we assume there is a synergistic effect of the combination of iMRI and 5-ALA. The latter is an operatordependent visualization procedure. Some areas of the tumor can be covered by blood or cotton patties. Those "invisible" tumor remnants can be detected by an operator-independent imaging like iMRI.

In $52.54 \%$ of our patients we achieved a GTR only after using iMRI, which demonstrates its importance. The rate of GTR was significantly higher after iMRI-guided additional resections compared with previously reported GTRs without 5-ALA and iMRI and with 5-ALA, but without iMRI. However, a comparison with other studies is limited. GTR is often not defined in the same way.

Table 7 Tumor volumes and duration of surgery as risk factors for SSI (two-sample $t$ test)

\begin{tabular}{|l|l|l|l|}
\hline Variables & SSI & No SSI & $p$ Value \\
\hline Preoperative tumor volume, $\mathbf{c m}^{3}$, mean value and sEM & $26.57 \pm 17.77$ & $29.58 \pm 27.94$ & 0.79 \\
\hline Duration of surgery, min, mean value and SEM & $246.25 \pm 19.16$ & $245.53 \pm 23.07$ & 0.953 \\
\hline
\end{tabular}

Abbreviations: SEM, standard error of the mean; SSI, surgical site infection. 
Mean EOR was $96.27 \%$ in our patient collective. EOR $>98 \%$ is known as a significant predictor for prolonged survival of GBM patients. ${ }^{1,2}$ Studies of 92 GBM patients showed that an EOR of $100 \%$ is related with a survival of 93 weeks, whereas survival decreases to 88.5 weeks if the EOR is between $75 \%$ and $99 \%$ and to 62.9 weeks, if the EOR is between $50 \%$ and $74 \%{ }^{25}$

In $69.6 \%$ of the GBM patients in our study, additional resections were performed after iMRI. The PPV that the Gdenhancing tissue detected by iMRI was astrocytic tumor, was $88.6 \%$. Another study showed a PPV of $64.3 \%$ for presence of tumor tissue after iMRI-guided resections and of $96 \%$ for 5 ALA-guided resection. ${ }^{26}$ This study however, was conducted with a low-field MRI. Therefore, PPV of iMRI in our study is higher than that of the low-field iMRI, but still lower than the PPV of 5-ALA. In general, it should be considered that the PPV is influenced by scar tissue in recurrent GBM and by surgical manipulation, so-called surgically induced enhancement. Sensitivity was $100 \%$ and specificity $70.6 \%$. Coburger et $\mathrm{al}^{27}$ reported a sensitivity of $41 \%$ and also a specificity of $70 \%$. It is difficult to compare the sensitivity of our study with the sensitivity in the study of Coburger et al, because these authors calculated the sensitivity by only considering World Health Organization grade IV tissue. . The sensitivity of $100 \%$ and the specificity of $70 \%$ in our study point to the risk of over diagnosing of tumor by iMRI. This phenomenon again might be linked to the fact that 28 (35.44\%) of our 79 patients had a recurrent GBM with scar tissue, and that also 8 patients (10.8\%) had a secondary GBM. Therefore, previous surgical or radiotherapeutic treatments have to be considered when interpreting iiMRI scans. The inclusion of those patients may be a limitation of our study, but on the other hand, inclusion of these patients represents a real life setting. Intraoperative magnetic resonance spectroscopy or perfusion-weighted MRI seem to be reasonable methods to reduce the problem of overdiagnosing.

Long-lasting procedures represent important risk factors for SSI. The prolongation of the surgery by iMRI and the fact that our MRI scanner is used for both diagnostic and intraoperative imaging made it necessary to examine the safety of our procedure. The mean iMRI scan time was 33.6 minutes. Including the transport and positioning of the patient, 55 minutes need to be scheduled. Indeed, the relative risk of operations lasting 2 hours causing infections is 12.6 and for those $>3$ hours is 24.3. These data were observed in 726 craniotomies and 663 spinal operations. ${ }^{28}$ However, duration of surgery was not a statistically significant variable in our analysis of possible risk factors for SSI.

Four SSIs were observed in 79 GBM operations in which iMRI was performed. Our SSI rate of $5.06 \%$ is within the normal range of neurosurgical procedures. The SSI rate in a collective of 1,007 patients with intracranial procedures performed without iMRI in our department was $5.45 \%$. The SSI rate in the group of recurrent gliomas with prior surgery, chemotherapy, and radiation therapy was $7.14 \%$. Therefore, the dual usage of the MRI scanner has not increased our SSI rate. Larger collectives composed of 1,181 and 3,042 patients showed SSI rates of $3.47 \%$ and $3.68 \%$, respectively. Some studies reported meningitis despite antibiotic prophylaxis

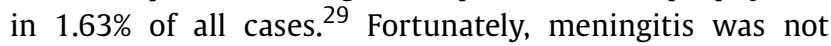
observed in our study. The causative agents of the SSIs in our study, included two cutaneous (one Staphylococcus epidermidis and one $S$. aureus), and two noncutaneous bacteria (Enterobacter cloacae and Pseudomonas aeruginosa). Cocci are responsible for $70.6 \%$ of all superficial and $64.5 \%$ of all deep SSIs. ${ }^{30}$ In addition to the decrease in infections caused by cutaneous bacteria, the frequent use of firstgeneration cephalosporins will likely increase SSIs caused by gram-negative bacteria. ${ }^{29}$

In our collective of the 29 patients in whom we also administered vancomycin and gentamicin intrathecally, an SSI rate of $0 \%$ was observed. In contrast, our cephalosporinonly group of 50 patients had an SSI rate of $8 \%$. However, that difference was not statistically significant. Other retrospective studies also claimed that the use of additional intrathecal antibiotic prophylaxis could be advantageous. Ragel et $\mathrm{al}^{18}$ determined the rate of SSIs with vancomycin combined with gentamicin to be $0.4 \%$, with gentamicin $5.4 \%$, and with standardized preoperative single-shot antibiotic prophylaxis $6.6 \%$. Taken these data and our findings into consideration, intrathecal antibiotic prophylaxis should be examined in future studies. ${ }^{18}$

Up to now iMRI is not universally established in neurosurgical departments because of the poor cost-benefit ratio if installed only for intraoperative use. Between July 2011 and June 2015, we performed 17,000 MRI scans for diagnostic use in addition to 250 intraoperative resection controls. Two to three iiMRI scans, $\sim 40$ diagnostic MRI scans for inpatients and 35 diagnostic scans for ambulatory patients were performed per week, allowing to use the iMRI economically.

\section{Conclusion}

There is obvious improved surgical success if iMRI together with 5-ALA are used. In particular, EOR was considerably higher in spite of the many GBMs located in eloquent areas. Intraoperative $\mathrm{Gd}$-enhancement is indicative of residual tumor and, therefore, can guide additional resections. Despite the dual use of our iMRI suite, the rate of SSIs was within the normal range for neurosurgical procedures. Consequently, sharing a high-field MRI for daily diagnostic use and intraoperative resection control is a safe and successful concept.

Ethical Approval

All procedures performed in studies involving human participants were in accordance with the ethical standards of the institutional and/or national research committee and with the 1964 Helsinki Declaration and its later amendments or comparable ethical standards.

\section{Informed Consent}

For this type of study, formal consent is not required. Institutional review board approval for the review of notes was obtained from Nottingham University Hospitals NHS Trust. 
Conflict of Interest

None declared.

\section{References}

1 Kuhnt D, Becker A, Ganslandt O, Bauer M, Buchfelder M, Nimsky C. Correlation of the extent of tumor volume resection and patient survival in surgery of glioblastoma multiforme with high-field intraoperative MRI guidance. Neuro Oncol 2011;13(12):1339-1348

2 Lacroix M, Abi-Said D, Fourney DR, et al. A multivariate analysis of 416 patients with glioblastoma multiforme: prognosis, extent of resection, and survival. J Neurosurg 2001;95(02):190-198

3 Soffietti R, Baumert BG, Bello L, et al; European Federation of Neurological Societies. Guidelines on management of low-grade gliomas: report of an EFNS-EANO Task Force. Eur J Neurol 2010;17 (09):1124-1133

4 Paterno' V, Fahlbusch R. High-field iMRI in transsphenoidal pituitary adenoma surgery with special respect to typical localization of residual tumor. Acta Neurochir (Wien) 2014;156(03): 463-474; discussion 474

5 Nimsky C, Ganslandt O, Cerny S, Hastreiter P, Greiner G, Fahlbusch R. Quantification of, visualization of, and compensation for brain shift using intraoperative magnetic resonance imaging. Neurosurgery 2000;47(05):1070-1079; discussion 1079-1080

6 Ohue S, Kumon Y, Nagato S, et al. Evaluation of intraoperative brain shift using an ultrasound-linked navigation system for brain tumor surgery. Neurol Med Chir (Tokyo) 2010;50(04):291-300

7 O'Keeffe AB, Lawrence T, Bojanic S. Oxford craniotomy infections database: a cost analysis of craniotomy infection. Br J Neurosurg 2012;26(02):265-269

8 López Pereira P, Díaz-Agero Pérez C, López Fresneña N, et al. Epidemiology of surgical site infection in a neurosurgery department. Br J Neurosurg 2017;31(01):10-15

9 Karhade AV, Cote DJ, Larsen AM, Smith TR. Neurosurgical infection rates and risk factors: a national surgical quality improvement program analysis of 132,000 patients, 2006-2014. World Neurosurg 2017;97:205-212

10 Dashti SR, Baharvahdat H, Spetzler RF, et al. Operative intracranial infection following craniotomy. Neurosurg Focus 2008;24(06):E10

11 Erman T, Demirhindi H, Göçer AI, Tuna M, Ildan F, Boyar B. Risk factors for surgical site infections in neurosurgery patients with antibiotic prophylaxis. Surg Neurol 2005;63(02):107-112; discussion 112-113

12 Barker FG II. Efficacy of prophylactic antibiotics for craniotomy: a meta-analysis. Neurosurgery 1994;35(03):484-490; discussion 491-492

13 Barker FG II. Efficacy of prophylactic antibiotics against meningitis after craniotomy: a meta-analysis. Neurosurgery 2007;60(05): 887-894; discussion 887-894

14 McClelland S III, Hall WA. Postoperative central nervous system infection: incidence and associated factors in 2111 neurosurgical procedures. Clin Infect Dis 2007;45(01):55-59

15 Abraham P, Lamba N, Acosta M, et al. Antibacterial prophylaxis for gram-positive and gram-negative infections in cranial surgery: a meta-analysis. J Clin Neurosci 2017;45:24-32

16 Liu W, Ni M,Zhang Y, Groen RJ. Antibiotic prophylaxis in craniotomy: a review. Neurosurg Rev 2014;37(03):407-414; discussion 414
17 Abdullah KG, Attiah MA, Olsen AS, Richardson A, Lucas TH. Reducing surgical site infections following craniotomy: examination of the use of topical vancomycin. J Neurosurg 2015;123(06): 1600-1604

18 Ragel BT, Browd SR, Schmidt RH. Surgical shunt infection: significant reduction when using intraventricular and systemic antibiotic agents. J Neurosurg 2006;105(02):242-247

19 Louis DN, Ohgaki H, Wiestler OD, et al. The 2007 WHO classification of tumours of the central nervous system. Acta Neuropathol 2007;114(02):97-109

20 Mangram AJ, Horan TC, Pearson ML, Silver LC, Jarvis WR; Hospital Infection Control Practices Advisory Committee. Guideline for prevention of surgical site infection, 1999. Infect Control Hosp Epidemiol 1999;20(04):250-278; quiz 279-280

21 Ma R, Watts C. Selective 5-aminolevulinic acid-induced protoporphyrin IX fluorescence in gliomas. Acta Neurochir (Wien) 2016;158(10):1935-1941

22 Roder C, Bisdas S, Ebner FH, et al. Maximizing the extent of resection and survival benefit of patients in glioblastoma surgery: high-field iMRI versus conventional and 5-ALA-assisted surgery. Eur J Surg Oncol 2014;40(03):297-304

23 Senft C, Bink A, Franz K, Vatter H, Gasser T, Seifert V. Intraoperative MRI guidance and extent of resection in glioma surgery: a randomised, controlled trial. Lancet Oncol 2011;12(11): 997-1003

24 Stummer W, Pichlmeier U, Meinel T, Wiestler OD, Zanella F, Reulen HJ; ALA-Glioma Study Group. Fluorescence-guided surgery with 5-aminolevulinic acid for resection of malignant glioma: a randomised controlled multicentre phase III trial. Lancet Oncol 2006;7(05):392-401

25 Keles GE, Anderson B, Berger MS. The effect of extent of resection on time to tumor progression and survival in patients with glioblastoma multiforme of the cerebral hemisphere. Surg Neurol 1999;52(04):371-379

26 Hauser SB, Kockro RA, Actor B, Sarnthein J, Bernays RL. Combining 5-aminolevulinic acid fluorescence and intraoperative magnetic resonance imaging in glioblastoma surgery: a histology-based evaluation. Neurosurgery 2016;78(04):475-483

27 Coburger J, Engelke J, Scheuerle A, et al. Tumor detection with 5-aminolevulinic acid fluorescence and Gd-DTPA-enhanced intraoperative MRI at the border of contrast-enhancing lesions: a prospective study based on histopathological assessment. Neurosurg Focus 2014;36(02):E3

28 Valentini LG, Casali C, Chatenoud L, Chiaffarino F, Uberti-Foppa C, Broggi G. Surgical site infections after elective neurosurgery: a survey of 1747 patients. Neurosurgery 2008;62(01):88-95; discussion 95-96

29 Korinek AM, Baugnon T, Golmard JL, van Effenterre R, Coriat P, Puybasset L. Risk factors for adult nosocomial meningitis after craniotomy: role of antibiotic prophylaxis. Neurosurgery 2006; 59:126-133

30 Korinek AM; Service Epidémiologie Hygiène et Prévention. Risk factors for neurosurgical site infections after craniotomy: a prospective multicenter study of 2944 patients. The French Study Group of Neurosurgical Infections, the SEHP, and the C-CLIN Paris-Nord. Neurosurgery 1997;41(05):1073-1079; discussion 1079-1081 